\title{
Absolute and convective instabilities in a one-dimensional Brusselator flow model
}

\author{
Kuznetsov, S.P.; Mosekilde, Erik; Dewel, G.; Borckmans, P.
}

Published in:

Journal of Chemical Physics

Link to article, DOI:

$10.1063 / 1.473763$

Publication date:

1997

Document Version

Publisher's PDF, also known as Version of record

Link back to DTU Orbit

Citation (APA):

Kuznetsov, S. P., Mosekilde, E., Dewel, G., \& Borckmans, P. (1997). Absolute and convective instabilities in a one-dimensional Brusselator flow model. Journal of Chemical Physics, 106(18), 7609-7616.

https://doi.org/10.1063/1.473763

\section{General rights}

Copyright and moral rights for the publications made accessible in the public portal are retained by the authors and/or other copyright owners and it is a condition of accessing publications that users recognise and abide by the legal requirements associated with these rights.

- Users may download and print one copy of any publication from the public portal for the purpose of private study or research.

- You may not further distribute the material or use it for any profit-making activity or commercial gain

- You may freely distribute the URL identifying the publication in the public portal

If you believe that this document breaches copyright please contact us providing details, and we will remove access to the work immediately and investigate your claim. 


\title{
Absolute and convective instabilities in a one-dimensional Brusselator flow model
}

\author{
Sergey P. Kuznetsov \\ Institute of Radio-Electronics, Russian Academy of Sciences, Zelenaya 38, Saratov 410019, Russia \\ Erik Mosekilde \\ Department of Physics, The Technical University of Denmark, 2800 Lyngby, Denmark \\ Guy Dewel and Pierre Borckmans \\ Service de Chimie Physique, Université Libre de Bruxelles, 1050 Brussels, Belgium
}

(Received 17 October 1996; accepted 7 February 1997)

\begin{abstract}
The paper considers a one-dimensional Brusselator model with a uniform flow of the mixture of reaction components. An absolute as well as a convective instability can arise for both the Hopf and the Turing modes. The corresponding linear stability analysis is presented and supported by the results of computer simulations of the nonlinear equations. Finally, the condition for spatially undamped tails (the Cherenkov condition) is obtained. This represents a new mechanism for pattern formation in chemical reaction-diffusion systems. (C) 1997 American Institute of Physics.

[S0021-9606(97)01818-7]
\end{abstract}

\section{INTRODUCTION}

Chemical reaction-diffusion systems can display instabilities of Hopf as well as of Turing type. ${ }^{1-3}$ The Hopf instability is associated with the onset of spatially uniform oscillations in time as a pair of complex conjugated eigenvalues of the Jacobian for the kinetic equations cross the imaginary axis. The Turing instability, ${ }^{4-6}$ on the other hand, arises as a single real eigenvalue crosses the imaginary axis, and the homogeneous steady state yields to the growth of spatial patterns of finite wavelength. In one dimension, this pattern has the form of a stripe structure. The condition for the Turing instability to arise before the Hopf instability is usually that the diffusion constants of the basic reagents are very different so that we can have short range activation and long range inhibition.

By contrast, for instance, to hydrodynamical instabilities, the Turing instability is characterized by an endogeneously controlled wavelength, determined by the rates of reaction and diffusion of the involved chemical species. A related phenomenon is the formation of Liesegang rings, ${ }^{7,8}$ where the chemical reaction-diffusion processes are coupled with physical precipitation. Recently, yet another instability has been studied ${ }^{9}$ that arises due to different flow velocities for the activator and inhibitor species.

Over the years a variety of different reaction-diffusion models have been investigated, including the Brusselator and Schnackenberg models, the Lengyel-Epstein model, and the Gray-Scott model. These studies have been directed, among other things, towards elucidating the formation of localized structures ${ }^{10}$ the emergence of secondary instabilities such as the Eckhaus and zig-zag instabilities, ${ }^{11}$ the competition of different modes in ramped systems, ${ }^{12}$ and the so-called spotmultiplication in systems exhibiting bistability. ${ }^{13}$ In all cases, the reaction-diffusion processes have been considered to take place in a resting medium.

The purpose of the present paper is to investigate a model of a reaction-diffusion system with uniform flow. As illustrated in Fig. 1, the reagents are assumed to be pumped continuously into the lengthy reaction space from the left. The mixture of species moves with a constant speed along the $x$-axis and leaves the reactor to the right. A setup of this type is clearly of relevance for many industrial processes, and it may also provide relatively simple conditions for the experimental investigation of chemical instabilities. We shall demonstrate that the pattern formation and spatio-temporal dynamics in the flow system depend crucially on the flow rate and that they can arise either in the form of convective or in the form of absolute instabilities.

An instability in a spatially extended system with translation invariance is termed absolute if a localized initial perturbation gives rise to growing amplitudes at all fixed points in space. This is illustrated in Fig. 2(a). The instability is called convective if the growing perturbation drifts in such a way that observation at a fixed point asymptotically shows an amplitude that decays towards zero [Fig. 2(b)]. The formal analysis of absolute and convective instabilities has been developed and widely applied in hydrodynamics, plasma physics, electronics and other fields. ${ }^{14-18}$ To the best of our knowledge, however, only a few studies have hinted at this approach in connection with reaction-diffusion systems. ${ }^{19-21}$ One of our aims is therefore to draw more attention to these useful concepts. We complete the analysis by discussing the Cerenkov condition for the emergence of spatially growing tails. This provides a new mechanism for pattern formation in chemical reaction-diffusion systems that can operate even with equal diffusion constants.

\section{LINEAR STABILITY ANALYSIS FOR THE SYSTEM WITH FLOW}

Let us consider the well-known Brusselator model as an example of a reaction-diffusion system that can produce Turing and Hopf instabilities. ${ }^{1,22}$ With proper choice of space 


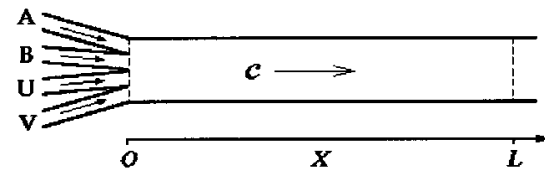

FIG. 1. Sketch of the reaction-diffusion flow model. The reagents $A$ (in excess), $B$ (in excess), $U$, and $V$ are pumped from the left edge. Their mixture moves with constant speed along the $x$-axis and leaves the reactor at the right edge.

and time units, and including the convective terms, this model is represented by the following kinetic equations:

$$
U_{t}+c U_{x}=A-B U-U+U^{2} V+\sigma U_{x x}
$$

and

$$
V_{t}+c V_{x}=B U-U^{2} V+V_{x x}
$$

with $U$ and $V$ denoting the concentrations of the interacting chemical species. $A$ and $B$ are externally controlled feed concentrations. $c$ is the flow rate, and $\sigma$ is the ratio of the two diffusion constants.

These equations obviously allow for the homogeneous steady state solution $U=A$ and $V=B / A$. Let us denote the input concentrations for the two reagents by $U_{0}$ and $V_{0}$. The left-hand side boundary conditions then become

$$
\left.U\right|_{x=0}=U_{0} \text { and }\left.V\right|_{x=0}=V_{0} .
$$

In most of the following analyses we shall assume the input concentrations to be equal to the steady state values $A$ and $B / A$.

Let us start with a spatially unbounded system and ask the question: How will the dynamics depend on the parameters $A, B, \sigma$, and $c$ for a localized small initial perturbation of the homogeneous steady state? We substitute

$$
U=A+u(x, t) \text { and } V=B / A+v(x, t)
$$

into the kinetic equations and assume $|u|,|v| \ll 1$. Then, in the linear approximation, we have

$$
\begin{aligned}
& u_{t}+c u_{x}=(B-1) u+A^{2} v+\sigma u_{x x}, \\
& v_{t}+c v_{x}=-B u-A^{2} v+v_{x x} .
\end{aligned}
$$

We suppose that the initial conditions are

$$
\left.u\right|_{t=0}=f(x) \text { and }\left.v\right|_{t=0}=g(x) \text {, }
$$

where the functions $f(x)$ and $g(x)$ decay rapidly for $x \rightarrow \pm \infty$. Following the standard approach, let us now perform a Laplace transformation of the linearized equations over the two independent variables $x$ and $t$. For $x$ we use the so-called two-sided version of the transformation. The relations for the forward and backward transforms are

$$
U_{s q}=\int_{0}^{\infty} e^{-s t} d t \int_{-\infty}^{\infty} u(x, t) e^{-q x} d x
$$

and

$$
u(x, t)=-\frac{1}{4 \pi^{2}} \int_{\beta-i \infty}^{\beta+i \infty} e^{s t} d s \int_{-i \infty}^{i \infty} U_{s q} e^{q x} d q,
$$
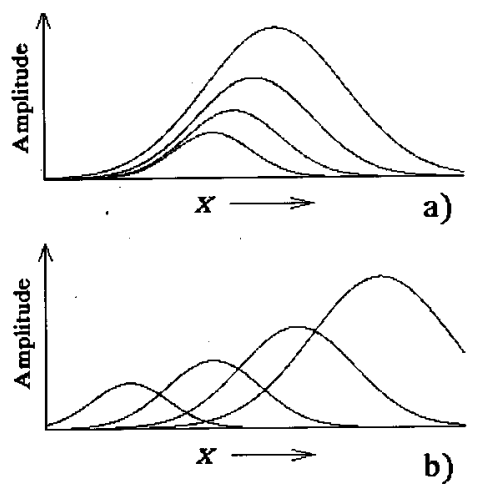

FIG. 2. Evolution of a localized perturbation in one-dimensional extended system in the cases of absolute (a) and convective (b) instabilities.

where $s$ and $q$ are complex variables. In formula (9) for the backward transformation, the integration contour in the $q$-plane is the imaginary axis. In the $s$-plane the contour is parallel to the imaginary axis and located to the right of all singularities of the integrand.

After this transformation, the kinetic equations read

$$
\left(s+c q-B+1-\sigma q^{2}\right) U_{s q}-A^{2} V_{s q}=F(q)
$$

and

$$
B U_{s q}+\left(s+c q+A^{2}-q^{2}\right) V_{s q}=G(q),
$$

where $F(q)$ and $G(q)$ are the transforms of $f(x)$ and $g(x)$. To reveal the presence of an instability and disclose its character, it is sufficient to consider one variable, say $U$. By solving the linear equations (10) and (11) we find $U_{s q}$ and then use the backward transformation (9) to obtain the following formal solution:

$$
\begin{aligned}
u(x, t)= & -\frac{1}{4 \pi^{2}} \int_{\beta-i \infty}^{\beta+i \infty} e^{s t} d s \\
& \times \int_{-i \infty}^{+i \infty} \frac{\left(s+c q+A^{2}-q^{2}\right) F(q)+A^{2} G(q)}{D(s, q)} e^{q x} d q,
\end{aligned}
$$

where the denominator

$D(s, q)=\left(s+c q-B+1-\sigma q^{2}\right)\left(s+c q+A^{2}-q^{2}\right)+A^{2} B$.

Under integration (12), $q$ runs along the imaginary axis. Hence, the solution $u(x, t)$ will contain components that grow with time if there exist purely imaginary values of $q$ for which the dispersion equation

$$
\left(s+c q-B+1-\sigma q^{2}\right)\left(s+c q+A^{2}-q^{2}\right)+A^{2} B=0
$$

has a root $s$ with positive real part. This is just the condition for instability. From the quadratic equation (13) we find the roots

$$
\begin{aligned}
s_{1,2}= & -c q-\frac{1}{2}\left\{1-B+A^{2}-\sigma q^{2}-q^{2}\right. \\
& \left. \pm\left[\left(1-B-A^{2}-\sigma q^{2}+q^{2}\right)^{2}-4 A^{2} B\right]^{1 / 2}\right\} .
\end{aligned}
$$


Substituting $q=i k$, we see that the onset of the instability does not depend on the flow rate (the parameter $c$ does not influence the real part of the roots).

It follows from Eq. (14) that the instability occurs in the range of wave numbers near $k=0$ for

$$
B>B_{H}=1+A^{2},
$$

and near $k= \pm A^{1 / 2} \sigma^{-1 / 4}$ for

$$
B>B_{T}=\left(1+A \sigma^{1 / 2}\right)^{2} .
$$

These expressions coincide with the well-known instability conditions for the Brusselator model without flow. ${ }^{22}$ The first type of instability is associated with a Hopf bifurcation in the spatially uniform system. This instability appears at the threshold as oscillations with frequency $\Omega=\operatorname{Im} s=A$ and wave number $k=0$. The second type is the Turing instability. For $c=0$ (no flow) it arises at zero frequency. For $c \neq 0$ the frequency at the threshold of the instability differs from zero, $\Omega=\operatorname{Im} s=c k$. This may be considered as a Doppler frequency shift; the Turing pattern with wave number $k$ moves in the laboratory frame with velocity $c$, producing the frequency $\Omega=c k$.

Now we have to determine whether the Hopf and Turing instabilities are absolute or convective. For this aim we must estimate carefully the integral (12) and study the asymptotic behavior of the solution $u(x, t)$ for $t \rightarrow \infty$ and $x=$ const.

Following Briggs ${ }^{16}$ and Bers, ${ }^{17}$ we may try to evaluate the outer integral by shifting the contour in the $s$-plane to the left, as far as possible without crossing any singularity of the integrand. If the system is stable, we can shift the contour in this way and place it entirely in the left half-plane. In this case the estimate for the solution yields $|u(x, t)|<$ const $\cdot e^{-\beta t}$, where the rate constant $\beta$ is the numerical value of the real part of that singularity which is closest to the imaginary axis in the $s$-plane.

If an instability occurs, a similar trick may be successful as long as the function under the outer integral allows analytic continuation in the $s$-plane (to the imaginary axis and beyond). The procedure of the analytic continuation corresponds to a deformation of the $q$-contour (the contour for the inner integral) that preserves the original bypass rules for the poles [zeros of the denominator $D(s, q)$ ]. If we can place the $s$-contour in the left half-plane while satisfying the above conditions, at any fixed position the perturbation $u(x, t)$ decays exponentially with time. Thus, the supposed instability can be only convective. Figure 3(a) shows the typical configuration of the integration contours in the $q$ and $s$-planes for this case. The dotted lines in the left diagram are trajectories of the poles in the $q$-plane traced while $s$ runs along the integration contour in the $s$-plane.

When trying to shift the $s$-contour to the left it may occur, however, that we find the $q$-contour pinched between two poles that arrive from opposite sides and merge [Fig. 3(b)]. This implies the presence of a branch point of the function $q(s)$. In other words, the dispersion equation has here a double root (with respect to $q$ ). The algebraic conditions for this situation to arise are
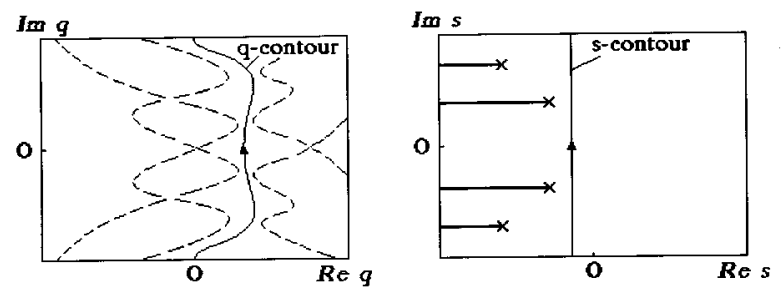

a)
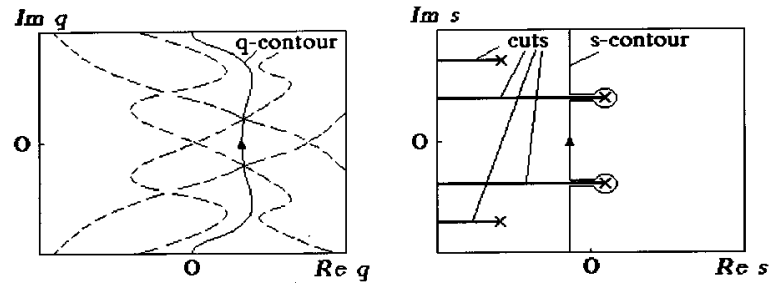

b)

FIG. 3. Contours for integration in the complex planes $q$ and $s$ in the absence (a) and presence (b) of an absolute instability. Dashed lines in the $q$-plane are trajectories of the poles (dispersion equation roots) that are traced while the variable $s$ runs along the contour in the $s$-plane. Branch points are marked by crosses, and the branch cuts are shown by solid lines.

$$
D(s, q)=0 \text { and } s^{\prime}(q)=0 .
$$

If the root of Eq. (17) $s=s_{b}=s\left(q_{b}\right)$ is located in the right half-plane, the instability is absolute. ${ }^{16,17}$ In this case the part of the $s$-contour running along the edges of the branch cut and around the branch point [Fig. 3(b), right] gives an increasing contribution to the integral

$$
u(x, t) \cong t^{-1 / 2} \exp \left(s_{b} t+q_{b} x\right) .
$$

[We recall that not all the branch points that may be obtained from Eq. (17) give rise to an absolute instability. Only those are relevant that appear due to merging of roots of the dispersion equation arriving from opposite sides of the $q$-contour when we decrease $\operatorname{Re} s$.]

Let us fix the parameters $\sigma$ and $A$ and study the change in character of the instability as we vary the remaining parameters $c$ and $B$. Following an idea proposed by Kuznetsov, ${ }^{23}$ we start with a search for the curves in the $(c, B)$ parameter plane that define the boundaries between absolute and convective instabilities. Then it will be sufficient to check the conditions of absolute instability at one representative point in each domain of the partition.

A boundary for the absolute instability may be associated with such a curve in the parameter plane where some branch point $s_{b}$ crosses the imaginary axis and enters into the right half-plane; the condition is

$$
\operatorname{Re} s\left(q_{b}\right)=0 \text {. }
$$

For $c=0$, the set of equations (17) can be solved analytically. This gives the branch points associated with the Hopf and Turing instabilities, respectively,

$$
\begin{aligned}
& q_{H}=0, \\
& s_{H}=\frac{1}{2}\left[-1+B-A^{2} \pm i \sqrt{4 A^{2}-\left(1-B+A^{2}\right)^{2}}\right],
\end{aligned}
$$




$$
q_{T}= \pm i\left[\frac{1-B-A^{2}+A(1+1 / \sigma)(\sigma B)^{1 / 2}}{1-\sigma}\right],
$$

and

$$
s_{T}=\frac{-1+B+\sigma A^{2}-2 A(\sigma B)^{1 / 2}}{1-\sigma} .
$$

The branch points cross the imaginary axis in the $s$-plane precisely at the values $B=B_{H}$ and $B_{T}$, as given by Eqs. (15) and (16). The points $\left(0, B_{H}\right)$ and $\left(0, B_{T}\right)$ are the starting points for two curves $H$ and $T$ in the $(c, B)$ parameter plane where the branch points have vanishing increment $\operatorname{Re} s$. To find these curves numerically, it is convenient to rewrite Eqs. (17) and (19) in the form

$$
D(\omega, q)=0, c D_{\omega}(\omega, q)+D_{q}(\omega, q)=0
$$

and

$$
\operatorname{Re}(\omega-c q)=0,
$$

where

$$
\omega=s+c q
$$

and

$$
D(\omega, q)=\left(\omega-B+1-\sigma q^{2}\right)\left(\omega+A^{2}-q^{2}\right)+A^{2} B .
$$

$D_{\omega}$ and $D_{q}$ denote partial derivatives of the function $D$. We substitute $q=q_{r}+i q_{i}, \omega=c q_{r}+i \omega_{i}$ and consider Eqs. (24)-(27) as a set of equations in four real unknowns $\left\{q_{r}, q_{i}, \omega_{i}, B\right\}$. This set may be solved numerically by Newton's method while step-by-step increasing the parameter $c$, starting from the points $\left(0, B_{H}\right)$ and $\left(0, B_{T}\right)$ where the solutions are known. In Fig. 4 we show the curves found for three different values of $A$ with $\sigma=0.25$.

In a similar manner we can trace simultaneously two branch points $s_{b, H}$ and $s_{b, T}$ in dependence on $c$ and $B$. By scanning the parameter region of interest and calculating the difference $\Delta=\operatorname{Re} s_{b, H}-\operatorname{Re} s_{b, T}$, we find the curves where $\Delta$ changes sign. They are shown in Fig. 4 as dotted lines marked by symbol $D$.

Dependent on $A$ and $\sigma$ we observe several possible situations for the mutual location of the boundary lines $H, T$, and $D$.

The threshold for the Hopf instability [see Fig. 4(a)], $B$ $=1+A^{2}$ falls below the Turing threshold. For small $c$, the curve $H$ is located below $T$. At some $c$, however, they cross each other. The curve $D$ consists of three pieces that meet at the "triple point" located above the curves $H$ and $T$. [For $c$ and $B$ corresponding to the triple point the saddle points responsible for the Hopf and Turing instabilities merge. With respect to $q$, Eqs. (17) here have two complex conjugate triple roots.] For $B<1+A^{2}$ the stationary spatially uniform equilibrium state is stable, and any perturbation can only decay. In the domain of instability $B>1+A^{2}$ we have six regions denoted by Roman numerals. Branch points with positive growth rates $\operatorname{Re} s$ are present in the regions II-VI.

Summarizing the results of the above analysis we have the following conclusions:

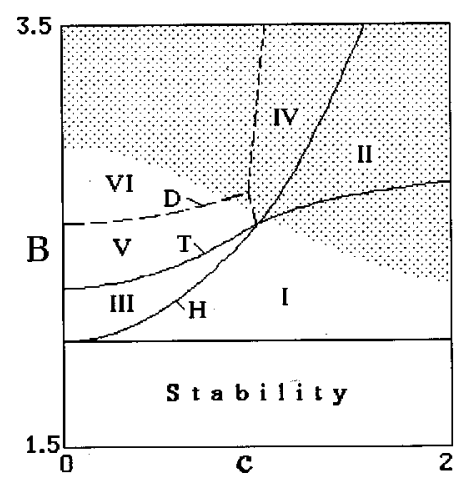

a)

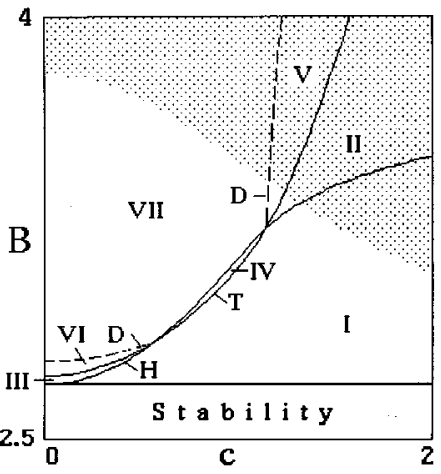

b)

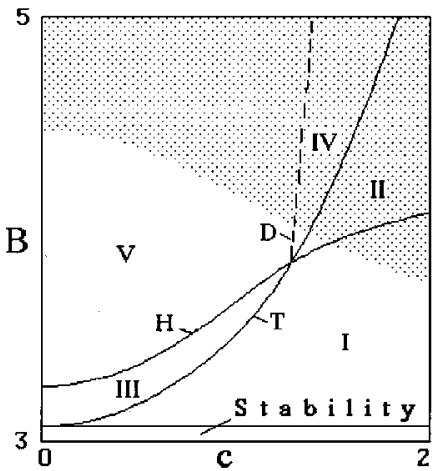

c)

FIG. 4. Domain in parameter plane for distinct behavior of small-amplitude perturbations of the spatially-uniform state for the Brusselator flow model; $\sigma=0.25$ and $A=1$ (a), 1.3 (b), and 1.5 (c). The curves $H$ and $T$ correspond to crossing the imaginary axis in $s$-plane by the branch point associated with Hopf of Turing instability, respectively. The dashed line $D$ is determined by requiring equal time increments at the branch points for the Hopf and Turing instabilities. There is only a convective instability in domains I and II. Numbers III and further mark the domains of absolute instability. Shading marks the parameter region where the edge perturbations become undamped in space.

(1) In regions I and II there is no absolute instability. In spite of the presence of a branch point with positive increment in region II, it does not satisfy the criterion; the merging roots arrive from the same side of the integration contour in the $q$-plane.

(2) In regions III and IV, an absolute instability of Hopf type takes place.

(3) In regions $\mathrm{V}$ and VI, both Hopf and Turing types of instability are absolute. In region $\mathrm{V}$, the Hopf instability has the larger growth rate, and vice versa in regions VI.

When increasing the parameter $A$ [see Fig. 4(b)], the 
triple point shifts downwards and crosses the intersection point of the curves $H$ and $T$. (From this moment a triple point degeneration occurs in the left half-plane of the $s$-variable, and it is no longer significant for the properties of the instabilities.) While the Hopf threshold remains lower than the Turing threshold $\left(B_{H}<B_{T}\right)$, the lines $H$ and $T$ have two intersections. The instability domain in Fig. 4(b) is partitioned into seven parts.

(a) In regions I and II there is no absolute instability.

(b) In region III, we have a Hopf absolute instability, while in regions IV and $\mathrm{V}$ a Turing absolute instability occurs. (We recall that in the case under consideration originally the Hopf threshold is lower! For sufficiently high flow rates, the order in which the Hopf and Turing absolute instabilities arise is interchanged as compared with the case of low flow rates.)

(c) In regions VI and VII, both Hopf and Turing types of instability are absolute. In region VI, the Hopf instability has the larger growth rate, and vice versa in region VII.

With further increase of $A$ [see Fig. 4(c)], the Turing threshold at zero velocity becomes lower than the Hopf threshold. Now, the instability domain is placed at $B>\left(1+A \sigma^{1 / 2}\right)^{2}$. For small $c$, the curve $T$ falls below $H$, and for larger $c$ they intersect. In the considered part of the parameter plane we see only one piece of the line $D$ emanating from the intersection point of the curves $H$ and $T$. The instability domain contains five regions.

(1) In regions I and II there is no absolute instability.

(2) In regions III and IV, we have Turing absolute instability.

(3) In region $V$, both the Hopf and Turing types of instability are absolute. The growth rate for the Turing instability is always the larger.

\section{NUMERICAL SIMULATION: ABSOLUTE INSTABILITY AND PATTERN FORMATION IN THE NONLINEAR FLOW SYSTEM}

It is interesting to return to the original nonlinear set of partial differential equations for the Brusselator flow model (1) and (2) and consider the observed large amplitude phenomena in the context of the linear analysis of the absolute and convective instabilities.

For the computer simulations we have used an implicit difference method of the second order. Typical step values $\Delta x$ and $\Delta t$ (in terms of the dimensionless position and time variables) were about 0.1 . The left edge boundary condition had the form (3) with constants $U_{0}$ and $V_{0}$ equal to the equilibrium concentrations $A$ and $B / A$, respectively. For sufficiently large length and flow rate $c>0$, the actual form of the boundary condition at $x=L$ is not relevant. In the computations we assumed free-end conditions,

$$
\left.U_{x}\right|_{x=L}=0,\left.\quad V_{x}\right|_{x=L}=0 .
$$

As initial conditions we used the steady state concentrations with small perturbations,

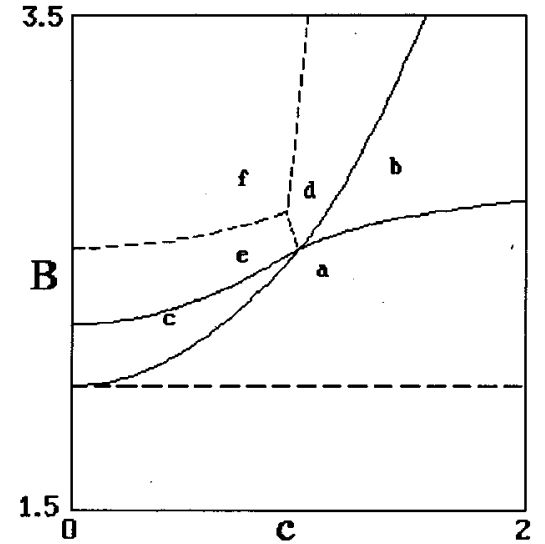

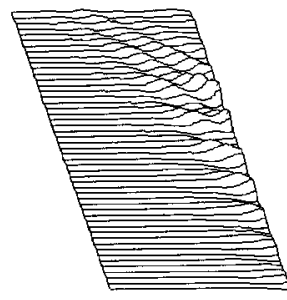

a)

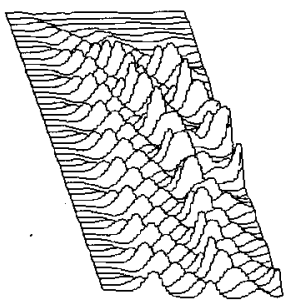

d)

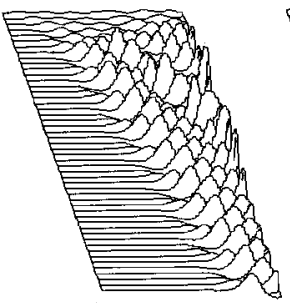

b)

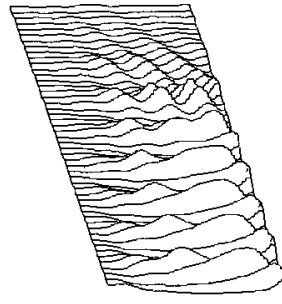

e)

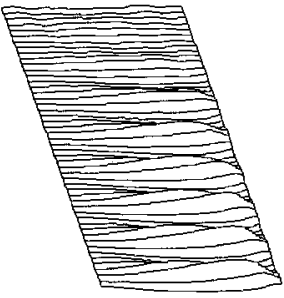

c)

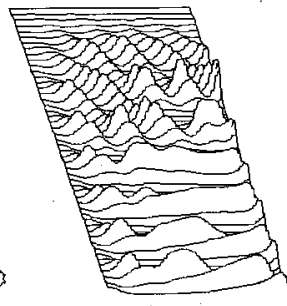

f)
FIG. 5. Results of numerical simulations of the Brusselator flow model with equilibrium input concentrations at the left edge of the reactor; $\sigma=0.25$, $A=1$. The spatio-temporal diagrams (a)-(f) show the dynamics of $U$ for the points in the $(c, B)$ parameter plane marked by the respective letters. The initial conditions are stationary homogeneous states with small-amplitude random perturbations. The dimensionless length of the reactor is $L=20$.

$$
U(m \Delta x, 0)=A+\xi_{m}, \quad V(m \Delta x, 0)=B / A+\eta_{m},
$$

where $\xi$ and $\eta$ are random numbers with zero mean value and a standard deviation of the order of 0.1 . $m \Delta x$ $(m=0,1,2, \ldots, N)$ denote points in the numerical grid.

Figure 5 shows examples of spatio-temporal dynamics observed in the distinct regions of parameter space discussed in the previous section, and Fig. 6 illustrates the changes in the pattern formation process that take place at the transition from convective to absolute instability for the Hopf (a) and the Turing (b) modes, respectively.

The left diagrams in Figs. 6(a) and 6(b) relate to the situation where the instability is present but not absolute. The wave perturbations are drifted down with the flow, and after a while the system approaches the spatially uniform steady state. The process appears like the motions of a front that separates spatial regions with and without oscillations. When decreasing the flow rate we observe that the front propagation becomes slower. Zero front velocity corresponds to the threshold of the absolute instability [the middle dia- 


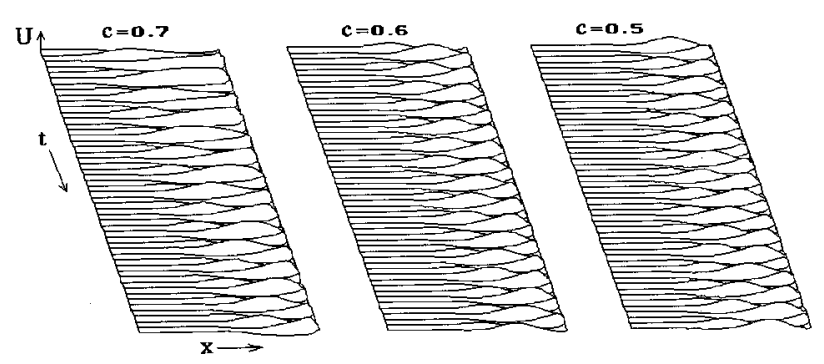

a) $6=0.25 ., A=1, B=2.206337$

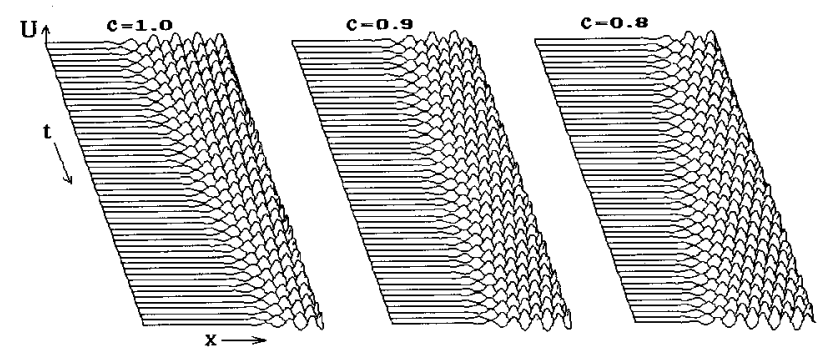

b) $\sigma=0.25 ., n=1.5 ., B=3.414392$

FIG. 6. Change in the nature of the pattern formation process at the transition from convective (left diagrams) to absolute instability (right diagrams) for Hopf (a) and Turing (b) modes. The flow speed is decreased from the left to the right pictures, other parameters are maintained constant. The middle diagrams correspond to the threshold of the absolute instability. Dimensionless length; (a) $L=50$, (b) $L=30$.

grams in Figs. 6(a) and 6(b)]. After crossing this boundary in parameter space we observe that the front velocity changes sign. If the fluctuations originally exist at the right edge of the system, we see the front propagate to the left, until, finally, the entire system is involved in the oscillation-wave dynamics (see diagrams to the right in Fig. 6).

Usually, one can easily distinguish patterns arising due to an absolute instability of Hopf or Turing type [see Fig. 6 and Figs. 5(c) and 5(d)]. In Figs. 5(e) and 5(f) we observe a competition between Turing and Hopf absolute instabilities (here the result is a dominance of the Hopf oscillations).

It is worth noticing the specific character of the transition associated with the boundary of the absolute instability in flow extended systems. In a system of large length this transition is accompanied by the sudden appearance of finite amplitude oscillations. However, this transition is reversible without hysteresis; during a slow backwards parameter tuning the oscillations disappear at the same parameter value where they arose, because the "bifurcation" consist in the reversion of the direction of propagation for the front separating oscillating and steady parts of the medium. Depending on this direction of propagation the bulk of the system appears to be involved or not to be involved in the oscillatory dynamics. In Fig. 7 we compare typical plots of amplitude vs parameter for usual soft and hard low-dimensional bifurcations $(a$ and $b)$ and for the transition under discussion $(c)$. (Actually, the amplitude jump becomes infinitely narrow only in the asymptotics of large length $L$.)

Figure 8 presents a number of spatio-temporal diagrams for a case where the parameter plane arrangement is similar to that of Fig. 4(b). However, to distinguish the characteristic

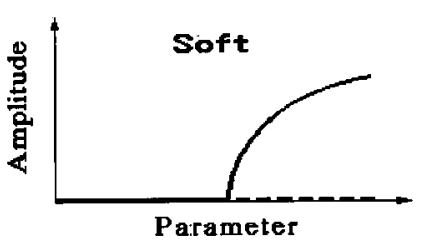

a)

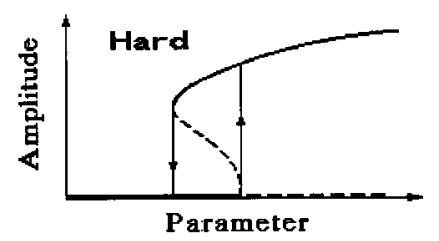

b)

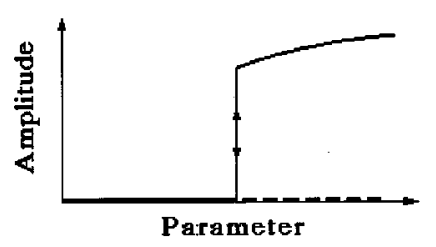

c)

FIG. 7. Amplitude dependence on a parameter; comparison of soft (a) and hard (b) bifurcations in low-dimensional systems with the transition from convective to absolute instability in extended system for the situation associated with reversal of the direction of front propagation (c).

regimes more clearly, we have used the values $\sigma=0.4$ and $A=0.25$. For small flow rates $c$, the onset of oscillations with increasing $B$ is associated with the appearance of a Hopf type absolute instability [Fig. 8(a)]. For larger values of $c$, the threshold of absolute instability for the Turing mode becomes lower than that of the Hopf mode. One can see the appearance and growth of the Turing patterns in Figs. 8(b)$8(\mathrm{~d})$. [The instability is absolute in cases (8b) and (8d) but not in (8c)]. This example illustrates the possibility of controlling the type of instability that occurs (as well as the type of the patterns generated) by tuning the flow rate.

\section{THRESHOLD FOR SPATIALLY UNDAMPED TAILS: CHERENKOV CONDITION}

Let us discuss a situation where the input concentrations of the reagents $U$ and $V$ differ from those for the homogeneous steady state. With a constant input flow of the reagents, a stationary spatial distribution may then arise and be stable. In this case, the concentration dependence on $x$ is governed by Eqs. (1) and (2) with vanishing time derivatives.

In the linear approximation we can analyze the spatial dependence for the concentration perturbations by means of the dispersion equation (13) with $s=0$,

$$
\left(c q-B+1-\sigma q^{2}\right)\left(c q+A^{2}-q^{2}\right)+A^{2} B=0 .
$$

For $c=0$, this equation becomes quadratic in $q^{2}$, and we can easily find all four roots; two with negative and two with 

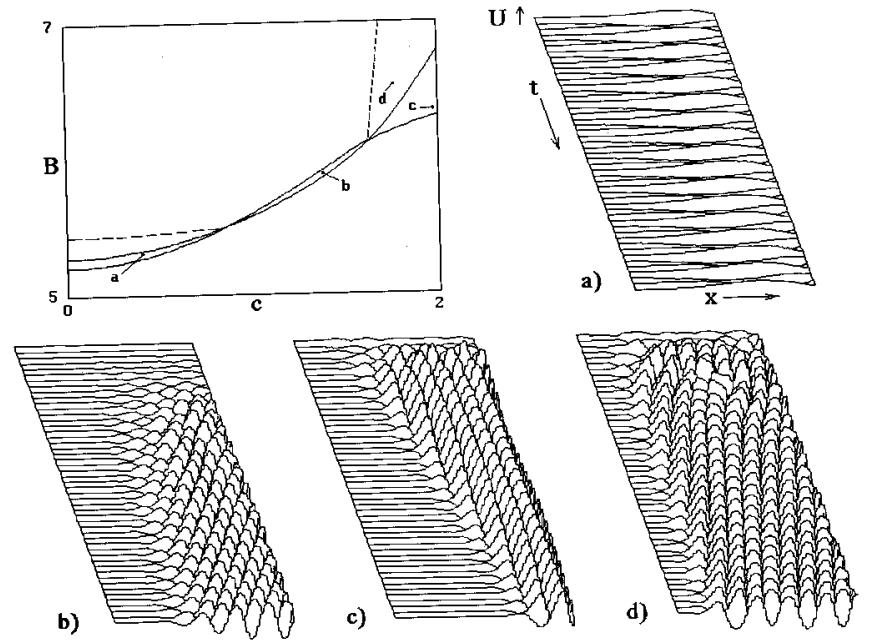

FIG. 8. Change of the nature of the absolute instability and pattern formation process in dependence of the flow velocity. For $\sigma=0.4, A=2.05$ in the $(c, B)$ parameter plane, the curves $H, T$, and $D$ are shown in a configuration similar to Fig. 4(b). The spatio-temporal diagrams illustrate the dynamics of $U$ at the points marked by the respective letters. While increasing $B$, an absolute instability of Hopf type appears first and gives rise to oscillations at small values of $c$ (a). For larger $c$, Turing patterns arise above the threshold of absolute instability.

positive real parts. For an unbounded system with constant perturbation at the origin the first two roots govern the spatial decay of the solution for $x>0$, and the second two roots the decay for $x<0$. Let us now increase the flow rate $c$. It may then occur that one of the roots responsible for the region $x>0$ crosses the imaginary axis and enters the right half-plane. This implies the onset of spatial amplification (convective instability) for a perturbation of zero frequency.

To find the associated condition of criticality, we insert $q=i k, k$ real, and separate the real and imaginary parts in Eq. (30). This gives the desired condition

$$
c^{2}=\left(1-B+\sigma A^{2}\right) /(1+\sigma)+A^{2}(1+\sigma) /\left(B-1-A^{2}\right)
$$

and defines a certain curve in the $(c, B)$ parameter plane. Above this curve the stationary wave perturbations are undamped in space. In Fig. 4 these regions are shaded.

We have performed a variety of computer simulations of the spatio-temporal dynamics for a case of nonequilibrium input concentration of $U$. The results are shown in Fig. 9. Below the boundary (31) the dynamics far from the left edge of the reactor remains the same as for equilibrium input concentrations [Figs. 9(a) and 9(b)]. However, near the left edge of the reactor we can see the formation of stationary spatial patterns. This tail decays in space, but becomes longer and longer as we move in the parameter plane towards the boundary given by Eq. (31). At this boundary and above, the conditions at the left edge influence the entire reactor space even in the case of very large length $L$. In the absence of the absolute instability, after the transient process we observe a stationary space periodic pattern [Fig. 9(c)]. This pattern forms the background state for instabilities that may take place in the parameter region under consideration [see Fig.

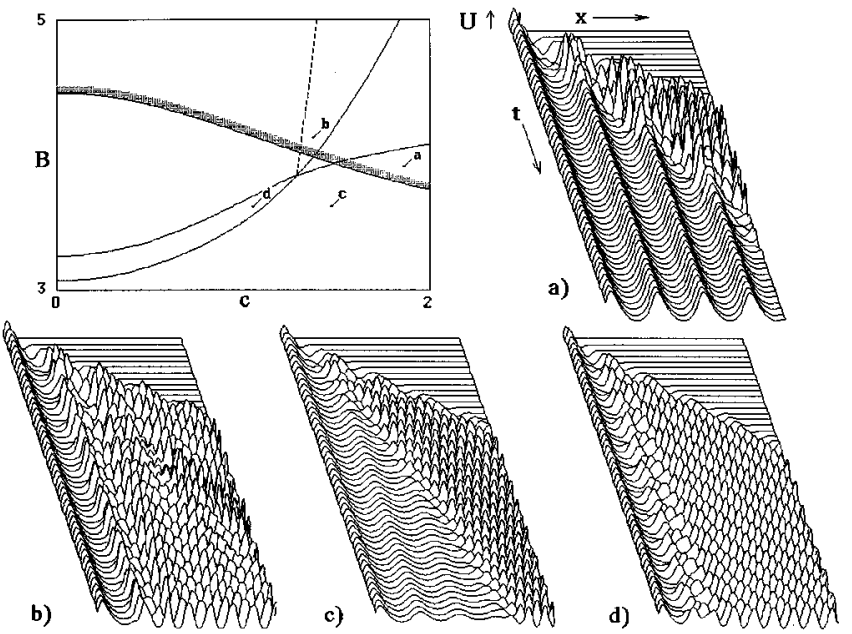

FIG. 9. Dynamics of the Brusselator flow model for a nonequilibrium input concentration $U_{x=0}=A+0.4$ and $V=B / A$. With $\sigma=0.25, A=1.5$ the curves $H, T$, and $D$ as well as the boundary of convective instability at zero frequency (marked by shading) are reproduced from Fig. 4(c). The spatiotemporal diagrams demonstrate the dynamics of $U$ at the points in the $(c, B)$ parameter plane marked by the corresponding letters. The initial conditions correspond to equilibrium concentrations of reagents. The dimensionless length of the system is $L=40$.

9(d)]. It is likely that the periodic pattern influences the location of the boundaries for the various instabilities. It is difficult to detect this effect in the numerical simulation, however.

In the frame moving with the flow the phenomenon may be interpreted as a "Cherenkov effect," the condition for emission of the wave from the moving local perturbation (the edge) is that the wave phase velocity and the velocity of the source be equal. Note that only the Hopf instability gives rise to such an effect. Actually, the spatial oscillations of the concentrations must be stationary in the laboratory frame, but an observer moving with the flow will se temporal oscillations with a nonzero frequency. The Turing instability in the moving frame arises just at zero frequency. It is interesting to notice that the Cherenkov effect may give rise to the appearance of standing structures even when the diffusion constants of the species are the same $(\sigma=1)$.

\section{CONCLUSION}

We have considered an extended one-dimensional reaction-diffusion model for the case of a moving mixture of reagents. The presence of a flow gives rise to a number of interesting phenomena. The Hopf and Turing instabilities can be either absolute or convective depending on the parameters. The transition to absolute instability is accompanied by a reversal of the direction of propagation for the front that separates spatial regions of steady state and wave oscillations in the medium. The properties of this transition differ from the bifurcations of low-dimensional systems; it is "hard", (the oscillations in a long system appear and disappear abruptly when adiabatically tuning the control parameter) but 
reversible (there is no difference in the "bifurcation" point during the forward and reverse tuning the parameter).

Depending on the control parameters it is possible to observe a local or a global influence of the boundary conditions on the spatio-temporal dynamics of the flow extended system. In the latter case the intrinsic instabilities will develop on the background of the pattern formed due to the presence of the boundary.

The situation that we have considered in this paper is directly amenable for experimental research on instabilities in reaction-diffusion systems. We expect that the various peculiarities of the spatio-temporal dynamics in flow systems can be observed under proper conditions, and that they will be significant for a variety of industrial processes. It should be noted, however, that the pinched point analysis may not apply to systems with destabilizing nonlinearities such as, for instance, in the case of a subcritical bifurcation. ${ }^{24}$

\section{ACKNOWLEDGMENTS}

The work was supported in part by the Russian Fund of Fundamental Researches (Grants Nos. 95-02-05818 and 9602-00717). G. Dewel and P. Borckmans are research associates with the FNRS (Belgium).

${ }^{1}$ G. Nicolis and I. Prigogine, Self-Organization in Nonequilibrium Systems (Wiley, New York, 1977).

${ }^{2}$ Oscillations and Traveling Waves in Chemical Systems, edited by R. J. Field and M. Burger (Wiley, New York, 1985).

${ }^{3}$ Chemical Waves and Patterns, edited by R. Kapral and K. Showalter (Kluwer Academic, The Netherlands, 1995).
${ }^{4}$ A. M. Turing, Trans. R. Soc. London, Ser. B 327, 37 (1952).

${ }^{5}$ V. Castets, E. Dulos, J. Boissonade, and P. De Kepper, Phys. Rev. Lett. 64, 2953 (1990); R. Rudovics, E. Dulos, and P. De Kepper, Phys. Scr. T 67, 43 (1996).

${ }^{6}$ Q. Ouyang and H. L. Swinney, Nature 352, 610 (1991); Q. Ouyang, Z. Noszticzius, and H. L. Swinney, J. Phys. Chem. 96, 6773 (1992).

${ }^{7}$ M. Flicker and J. Ross, J. Chem. Phys. 60, 3458 (1974).

${ }^{8}$ J. Ross, A. P. Arkin, and S. C. Müller, J. Phys. Chem. 99, 10417 (1995).

${ }^{9}$ A. B. Rovinsky and M. Menzinger, Phys. Rev. Lett. 69, 1193 (1992); 70, 778 (1993).

${ }^{10}$ O. Jensen, V. O. Pannbacker, E. Mosekilde, G. Dewel, and P. Borckmans, Phys. Rev. E 50, 736 (1994).

${ }^{11}$ V. Dufiet and J. Boissonade, Physica A 188, 158 (1992).

${ }^{12}$ P. Borckmans, A. De Wit, and G. Dewel, Physica A 188, 137 (1992).

${ }^{13}$ J. E. Pearson, Science 261, 189 (1993).

${ }^{14}$ P. A. Sturrock, Phys. Rev. 112, 1488 (1958).

${ }^{15}$ L. D. Landau and E. M. Lifshitz, Fluid Mechanics (Pergamon, Oxford, 1959).

${ }^{16}$ R. J. Briggs, Electron Stream Interaction with Plasma (MIT, Cambridge, 1964).

${ }^{17}$ A. Bers, in Handbook of Plasma Physics, edited by A. A. Galaev and R. N. Sudan (North-Holland, Amsterdam, 1983), p. 451.

${ }^{18}$ A. B. Mikhailovsky, Theory of Plasma Instabilities (Teorija Plazmennykh Neustoichivostei) (Atomizdat, Moscow, 1975), Vol. 1.

${ }^{19}$ R. Friedrich, M. Bestehorn, and H. Haken, Int. J. Mod. Phys. 4, 365 (1990).

${ }^{20}$ S. Ponce Dawson, A. Lawniczak, and R. Kapral, J. Chem. Phys. 100, 5211 (1994).

${ }^{21}$ P. Borckmans, G. Dewel, A. De Wit, and D. Walgraef, in Chemical Waves and Patterns, edited by R. Kapral and K. Showalter (Kluwer Academic, 1995), p. 323.

${ }^{22}$ H. Kidachi, Prog. Theor. Phys. 63, 1152 (1980).

${ }^{23}$ A. P. Kuznetsov, Sov. Tech. Phys. Lett. 8, 408 (1982).

${ }^{24}$ J. M. Chomaz, Phys. Rev. Lett. 69, 1931 (1992). 